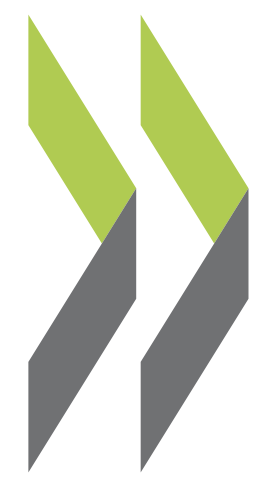

OECD Development Co-operation Working Papers No. 11

\author{
Les garanties \\ pour le développement
}

\author{
Mariana Mirabile, \\ Julia Benn,
}

Cécile Sangaré 


\section{Les garanties pour le développement}

Mariana Mirabile, Julia Benn, et Cécile Sangaré

Organisation de Coopération et de Développement Économiques, France 


\section{Résumé}

Ce document de travail présente les résultats d'une enquête sur les garanties pour le développement menée dans le cadre des travaux du CAD de l'OCDE destinés à améliorer les statistiques sur le financement extérieur du développement après 2015. Actuellement, il n'existe pas de données complètes et comparables au niveau international sur les garanties pour le développement et le volume des fonds qu'elles mobilisent. Cette enquête visait à combler ce déficit d'information en estimant le volume des apports du secteur privé aux pays en développement mobilisés au moyen de mécanismes de garantie. Les garanties accordées à des fins de développement ont mobilisé plus de 15 milliards USD d'apports du secteur privé vers/dans les pays en développement au cours de la période étudiée (2009-2011). Ce rapport analyse les données provenant de l'enquête (notamment par secteur et par pays), comprend une réflexion sur la façon d'appréhender au niveau international le montant mobilisé par les garanties et présente des commentaires sur le ratio de levier en tant que mesure de l'efficience du financement du développement.

Cet ouvrage est publié sous la responsabilité du Secrétaire général de l'Organisation de Coopération et de Développement Économiques (OCDE). Les opinions et les interprétations exprimées ne reflètent pas nécessairement les vues de l'OCDE ou des gouvernements de ses pays membres.

Ce document et toute carte qu'il peut comprendre sont sans préjudice du statut de tout territoire, de la souveraineté s'exerçant sur ce dernier, du tracé des frontières et limites internationales, et du nom de tout territoire, ville ou région. 


\section{Table of Contents}

I. Introduction

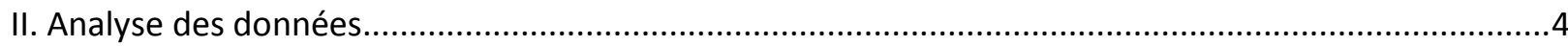

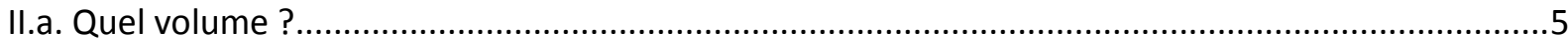

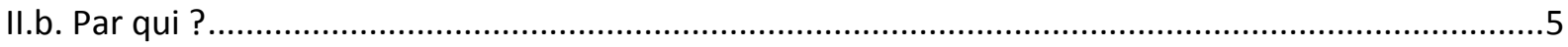

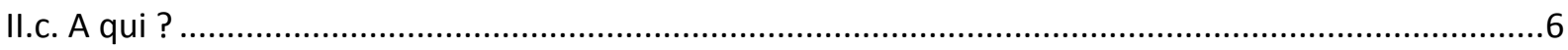

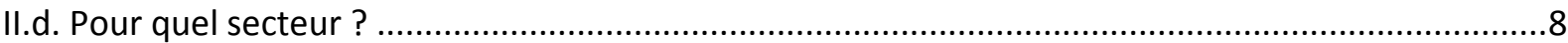

II.e Quelles sont les principales caractéristiques des garanties émises à des fins de développement ?10

III. Mesurer l'effet catalyseur des garanties : une première réflexion ....................................................11

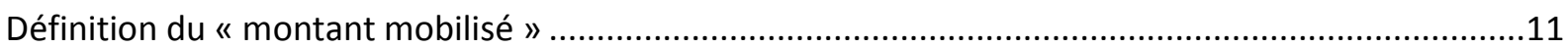

III.b. Montant mobilisé vs ratio de levier - difficultés posées par le calcul de l'effet de levier.............14

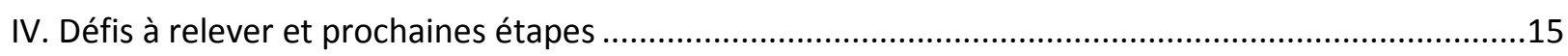

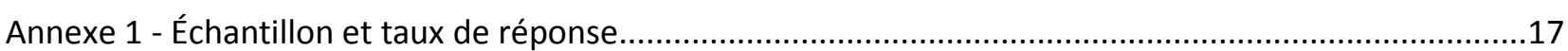

Annexe 2 - Pays bénéficiant de garanties à long terme ......................................................................20

Annexe 3 - Calculer le ratio de levier : exemple concret, données requises et méthodologies possibles ...21 


\title{
LES GARANTIES POUR LE DÉVELOPPEMENT
}

\author{
Étude réalisée par le Groupe de travail du CAD sur les statistiques du financement du développement \\ (GT-STAT) du Comité d'aide au développement (CAD) de l'OCDE
}

\section{Introduction}

Le Comité d'aide au développement (CAD) de l'OCDE collecte et publie des données sur les apports de ressources extérieures, autrement dit sur les ressources financières, les biens ou les services allant des pays développés vers les pays en développement. Lors de sa réunion à haut niveau en décembre 2012, le CAD a reçu pour mandat d'examiner dans une perspective nouvelle et plus large le financement du développement et d'améliorer les statistiques sur le financement extérieur du développement autre que l'aide publique au développement (APD).

Actuellement, il n'existe pas de données complètes et comparables au niveau international sur les garanties pour le développement et le volume des fonds qu'elles mobilisent : les garanties ne sont pas prises en compte dans le cadre statistique du CAD, ni de façon plus générale dans les statistiques financières internationales. L'enquête sur les garanties pour le développement, réalisée dans le cadre du nouveau mandat du CAD, visait à contribuer à combler ce déficit d'information ${ }^{1}$.

Pour les besoins de l'enquête, les " garanties pour le développement » ont été définies comme étant les garanties accordées dans l'objectif principal de favoriser le développement économique et l'amélioration du niveau de vie des pays en développement. L'enquête s'est appuyée sur un questionnaire envoyé aux institutions de financement du développement (IFD), aux organismes d'aide et aux ministères des affaires étrangères dans 24 pays du CAD et dans 12 pays non membres du CAD, ainsi qu'à 17 institutions financières internationales (IFI) (voir annexe 1$)^{2}$.

L'enquête avait pour principal objet d'évaluer le volume des apports du secteur privé aux pays en développement mobilisés au moyen de mécanismes de garantie au cours de la période 2009-2011. Elle visait également à : i) déterminer la faisabilité, à l'avenir, de collecter des données qualitatives et quantitatives sur les mécanismes de garantie, dans le cadre de la notification au CAD de données statistiques sur le financement extérieur du développement; et ii) nourrir la réflexion en cours dans différents espaces de dialogue sur la façon de mesurer l'effet de levier des divers instruments utilisés pour financer le développement.

Ce rapport est structuré comme suit. La section II rappelle les termes clés et présente les principaux résultats de l'analyse des données, notamment une estimation du montant mobilisé par les mécanismes de garantie. La section III expose les raisons ayant présidé au choix de la définition du "montant mobilisé ", compare les modalités de calcul de ce montant et celles du ratio de levier puis présente des commentaires sur ce dernier comme mesure de l'efficience du financement du développement. Pour finir, la section IV résume les questions qui se posent et les prochaines étapes.

Cette publication vise à encourager la réflexion sur le rôle et la mesure des garanties dans le cadre du financement du développement de l'après 2015. Les commentaires et propositions au Secrétariat sur ce sujet sont les bienvenus et peuvent être envoyés à l'adresse suivante : dac.contact@oecd.org.

1. Les informations détaillées recueillies dans le cadre du présent rapport au niveau des garanties individuelles sont confidentielles. Les résultats sont donc présentés à un niveau agrégé.

2. Les garanties accordées par les organismes de crédit à l'exportation ont été exclues, car elles n'ont pas le développement pour objectif principal. 


\section{Analyse des données}

Cette section présente les principaux résultats de l'enquête. Les données couvrent plus de 1000 garanties à long terme ${ }^{3}$ accordées par 14 pays et organisations (voir tableau A2). Les termes clés utilisés pour la collecte et l'analyse des données de l'enquête sont rappelés dans l'encadré 1. Quelques caractéristiques de garanties à court terme sont exposées dans l'encadré 2.

\section{Encadré 1 - Définition des termes clés}

Le terme garantie désigne un accord juridiquement contraignant selon lequel le garant accepte de payer tout ou partie du montant dû sur un prêt, une prise de participation ou un autre instrument en cas de nonpaiement par le débiteur ou de perte de valeur dans le cas d'un investissement.

Les garanties pour le développement sont celles accordées dans l'objectif principal de favoriser le développement économique et l'amélioration du niveau de vie des pays en développement (autrement dit celles motivées par des considérations de développement). L'enquête a été réalisée selon une " approche institutionnelle " au sens où seules les institutions investies d'une mission en matière de développement ont été incluses dans l'échantillon. ${ }^{4}$

Le montant mobilisé par une garantie est la valeur nominale totale de l'instrument (par exemple, prêt, prise de participation) auquel la garantie est attachée, quelle que soit la part de cette valeur couverte par la garantie (4 millions USD dans le graphique 1).

L'exposition brute est la somme totale que le garant versera à l'investisseur si le risque couvert se matérialise, indépendamment de la réassurance (3.6 millions USD dans le graphique 1).

L'exposition nette est l'exposition brute moins la somme que le garant recouvrera par le jeu de la réassurance (1.8 million USD dans le graphique 1 ).

Graphique 1 - Montant mobilisé, expositions brute et nette tels que définis dans l'enquête (exemple)

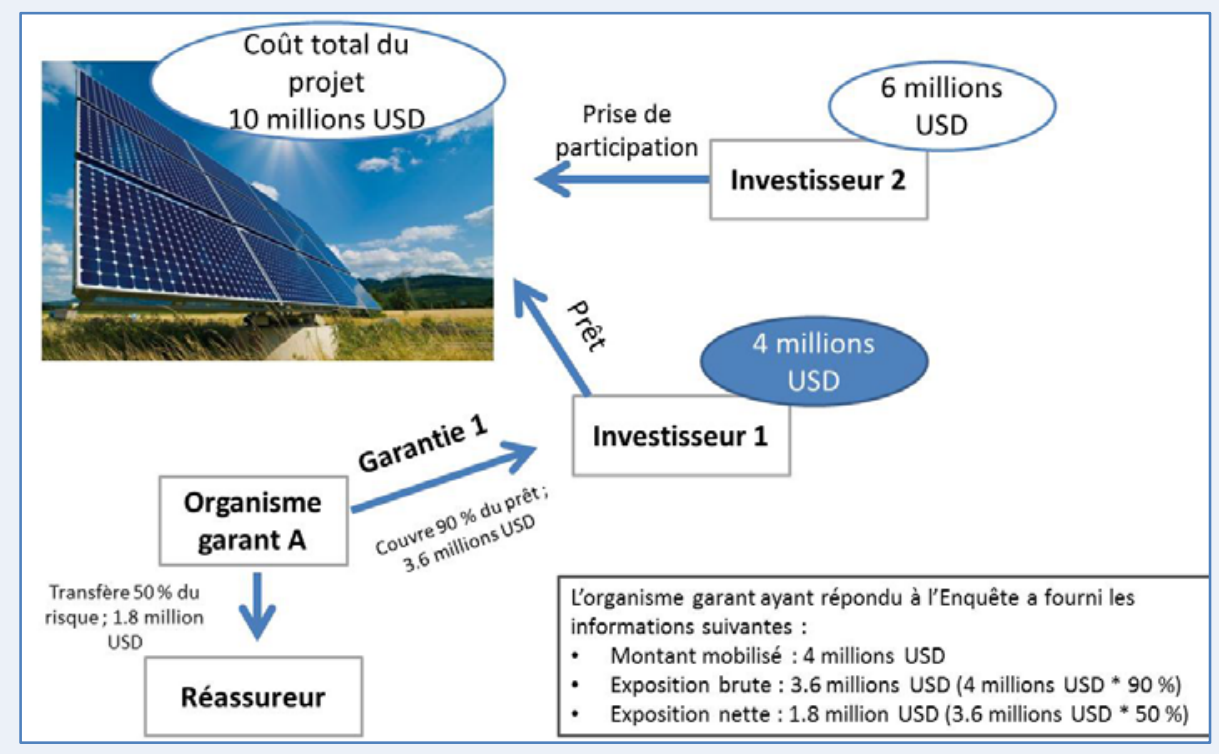

3. Les garanties à long terme couvrent les risques pendant un an ou plus. Les données sur les garanties à court terme ont été collectées à un niveau agrégé pour des questions de confidentialité et, de ce fait, ne pouvaient pas être incluses dans l'analyse générale des données.

4

Un membre a cependant notifié plusieurs garanties émises par l'organisme de crédit à l'exportation au nom du ministère des Affaires étrangères. 


\section{II.a. Quel volume?}

Les garanties pour le développement - accordées par les gouvernements des pays donneurs du CAD (organismes d'aide et IFD) et les IFI - ont mobilisé 15.3 milliards USD auprès du secteur privé à des fins de développement de 2009 à 2011.

Le graphique 2 montre les montants que les mécanismes de garantie ont permis de mobiliser auprès du secteur privé à des fins de développement et le risque pris par le garant - mesuré par l'exposition nette - pour la période allant de 2009 à $2011^{5}$.

Graphique 2 - Montant mobilisé et exposition nette (milliards USD)

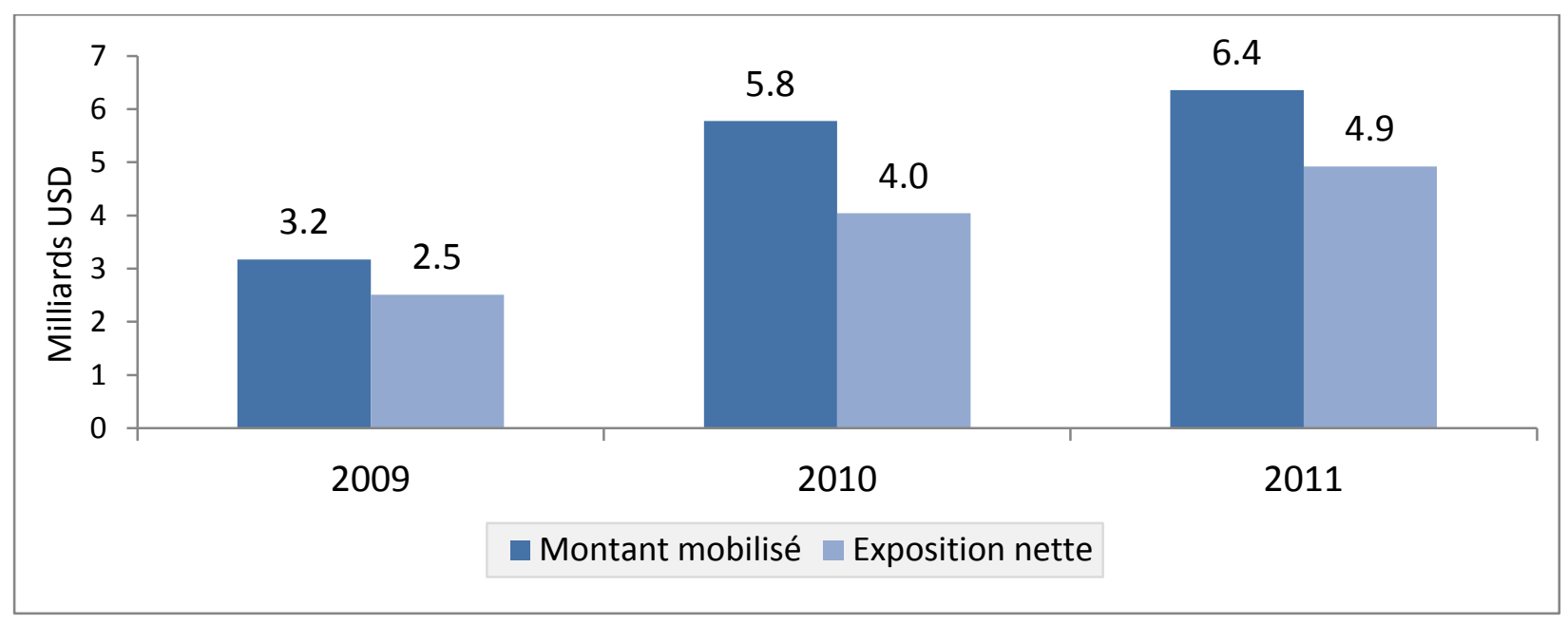

Le montant mobilisé par les garanties - c'est-à-dire les financements du secteur privé à l'appui des projets dans les pays en développement - a doublé, passant de 3.2 milliards USD en 2009 à 6.4 milliards en 2011. L'exposition nette moyenne s'élevait à $70 \%$ du montant mobilisé.

Le volume des ressources mobilisées pour le développement par le jeu des mécanismes de garantie reste modeste par rapport à l'ensemble du financement du développement. En 2011, les garanties motivées par des considérations de développement couvraient des activités d'un montant évalué à 6.4 milliards USD, soit environ $12 \%$ de l'aide programmable par pays ( 54.8 milliards USD) et moins de $1 \%$ des apports privés internationaux. Cependant, le recours aux garanties pourrait apparemment être considérablement étendu : plusieurs donneurs n'ont pas encore mis en place de programmes de garantie, et ceux qui offrent des garanties élargissent actuellement leur utilisation.

\section{II.b. Par qui ?}

Plus de $50 \%$ du montant mobilisé sont garantis par des organismes multilatéraux (IFI).

Les résultats de l'enquête montrent que les IFI utilisent davantage les garanties à des fins de développement que leurs homologues bilatérales (IFD), à l'exception de I'OPIC, qui contribuait à $65 \%$ du montant total mobilisé par les IFD. Plusieurs institutions bilatérales ont déclaré que, comme elles sont légalement tenues de proposer uniquement des produits financiers éligibles à l'APD, elles ne pouvaient inclure les garanties dans

5. L'exposition brute a été prise comme valeur indicative du montant mobilisé quand ce dernier n'était pas disponible. Pour quelques garanties, on ne dispose pas de données sur l'exposition nette. 
leur portefeuille. Les garanties ne sont pas comptabilisées dans l'APD, car elles ne constituent pas un apport financier.

\section{$15 \%$ des ressources mobilisées par les garanties sont domestiques.}

Le graphique 3 montre l'origine des apports privés mobilisés et leur montant. II est intéressant de constater que les garanties ont aussi mobilisé un important volume de ressources dans les pays en développement: $15 \%$ des ressources mobilisées par les garanties ( 2.3 milliards USD) en 2009-2011 sont domestiques.

\section{Graphique 3 - Montant mobilisé par origine des fonds}

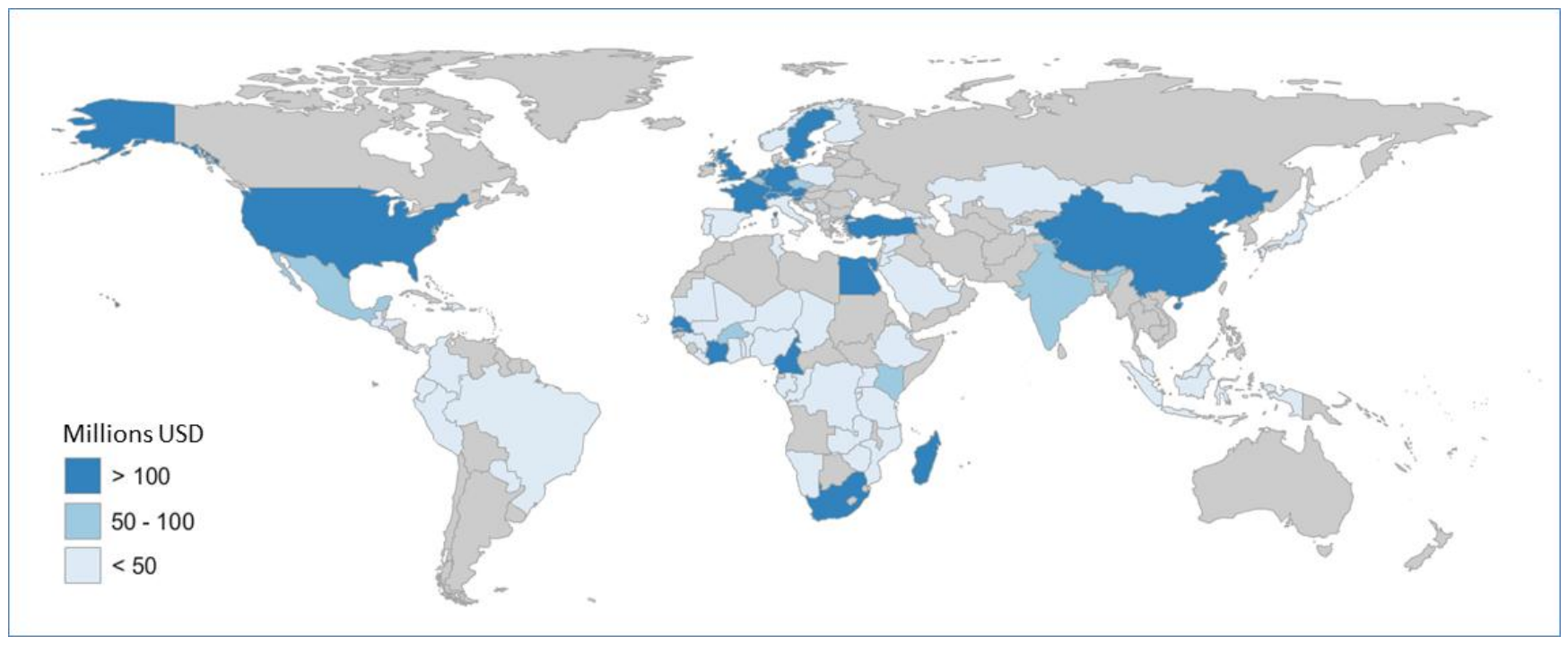

II.c. A qui ?

Plus de $50 \%$ des ressources mobilisées par les garanties ont bénéficié aux pays à revenu intermédiaire de la tranche supérieure.

En termes de nombre de garanties accordées (graphique 4, à gauche), presque $40 \%$ des contrats établis l'ont été au profit des pays les moins avancés (PMA). Cependant, en termes de montant, plus de la moitié de l'ensemble des ressources mobilisées est allée à des pays à revenu intermédiaire de la tranche supérieure (graphique 4, à droite). Ces chiffres indiquent que les contrats sont d'un volume sensiblement plus faible dans les PMA. L'exposition moyenne au risque (non représentée dans le graphique 4) ne varie pas de manière significative d'un groupe de revenu à l'autre. 
Graphique 4 - Montant mobilisé par groupe de revenu des bénéficiaires
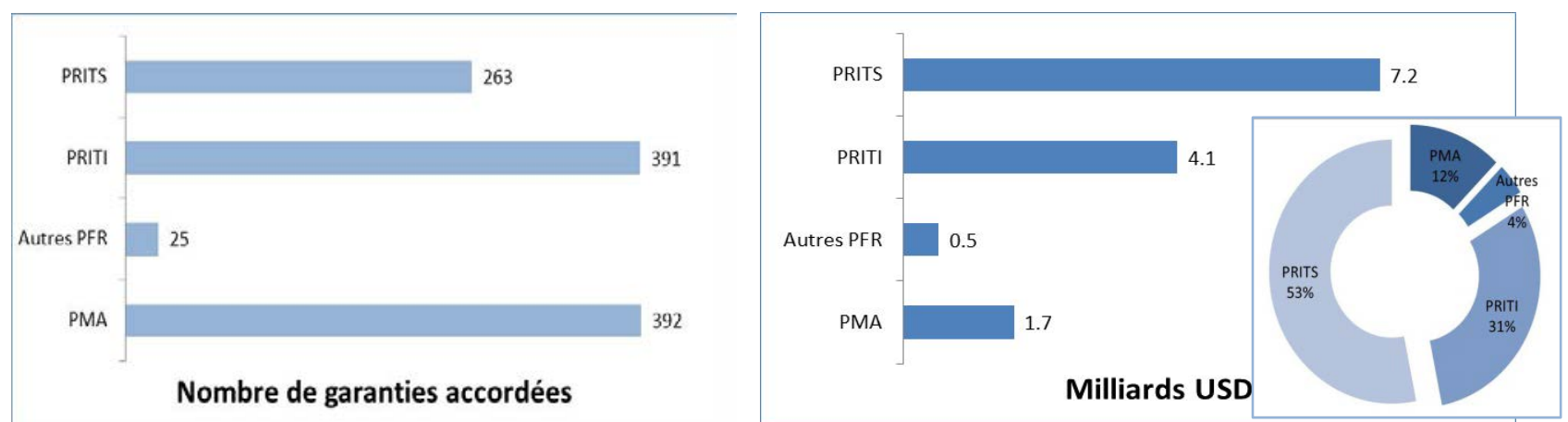

PMA : pays les moins avancés ; Autres PFR : autres pays à faible revenu ; PRITI : pays à revenu intermédiaire de la tranche inférieure ; PRITS : pays à revenu intermédiaire de la tranche supérieure.

\section{L'Afrique est la région qui a le plus bénéficié des garanties.}

La région ayant le plus bénéficié des garanties est l'Afrique, suivie de l'Asie et de l'Europe de l'Est (respectivement $41 \%, 24 \%$ et $22 \%$ du montant mobilisé total). Plus d'un tiers des ressources mises à disposition en Afrique sont allées à des PRITS - en particulier au Botswana, à la Tunisie et à l'Afrique du Sud et un autre tiers a bénéficié à des PMA et à d'autres PFR. En termes de nombre de contrats, plus de 650 garanties ont été émises en Afrique, 201 en Asie, 74 en Europe et 143 en Amérique Latine. Les contrats émis en Afrique étaient sensiblement plus modestes que ceux émis dans les autres régions. La liste des pays bénéficiaires figure dans l'annexe 2 .

Graphique 5 - Régions bénéficiant des ressources mobilisées par les garanties (milliards USD)

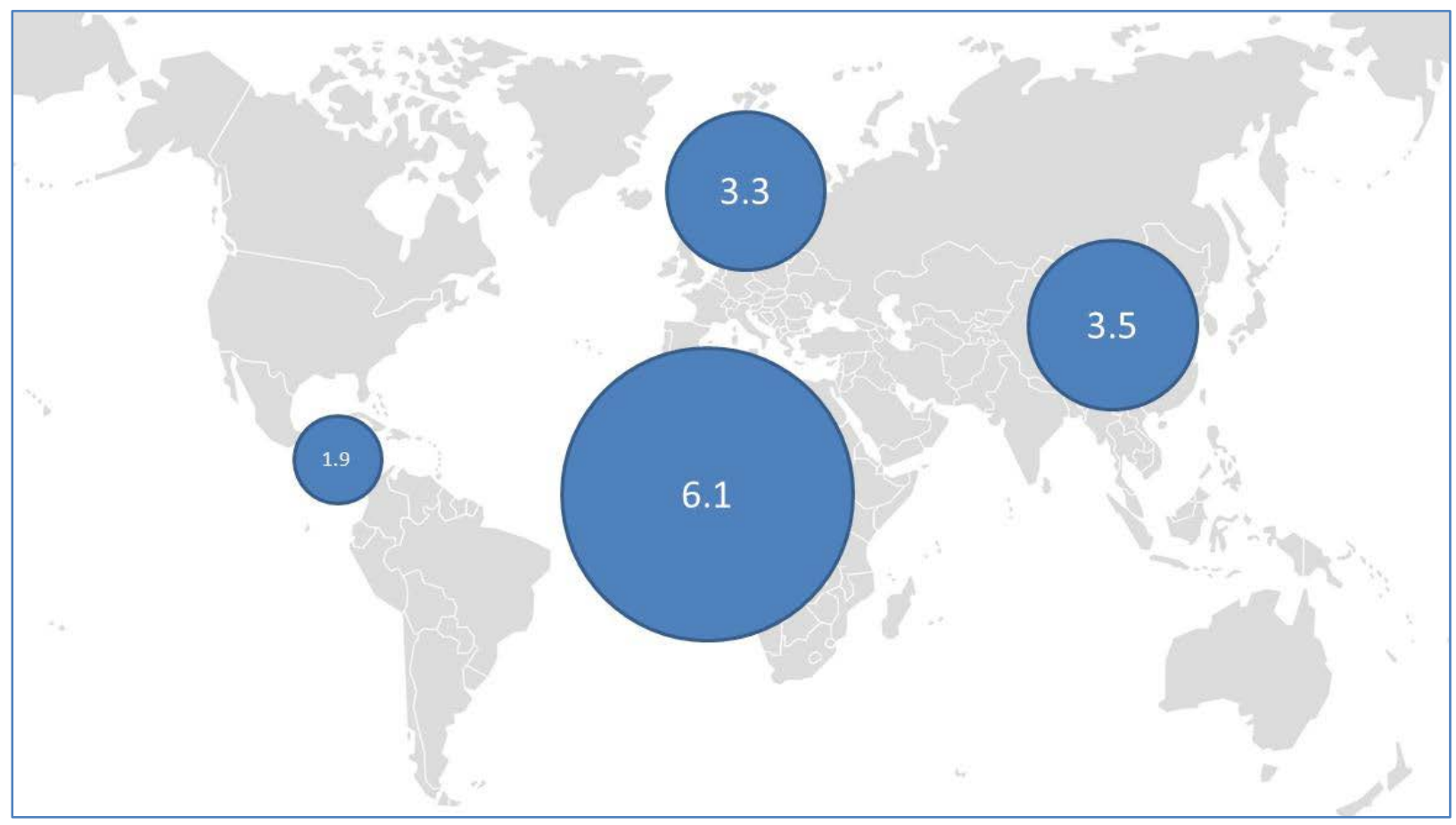




\section{II.d. Pour quel secteur?}

\section{Le secteur des services bancaires et financiers a le plus bénéficié des garanties.}

Le graphique 6 montre les cinq premiers secteurs ayant bénéficié des garanties ${ }^{6}$. Les garanties ont principalement été utilisées pour les services bancaires et financiers, par exemple, les lignes de crédit pour la microfinance, l'amélioration de la structure du portefeuille et le refinancement.

\section{Graphique 6 - Les cinq premiers secteurs bénéficiant des garanties}

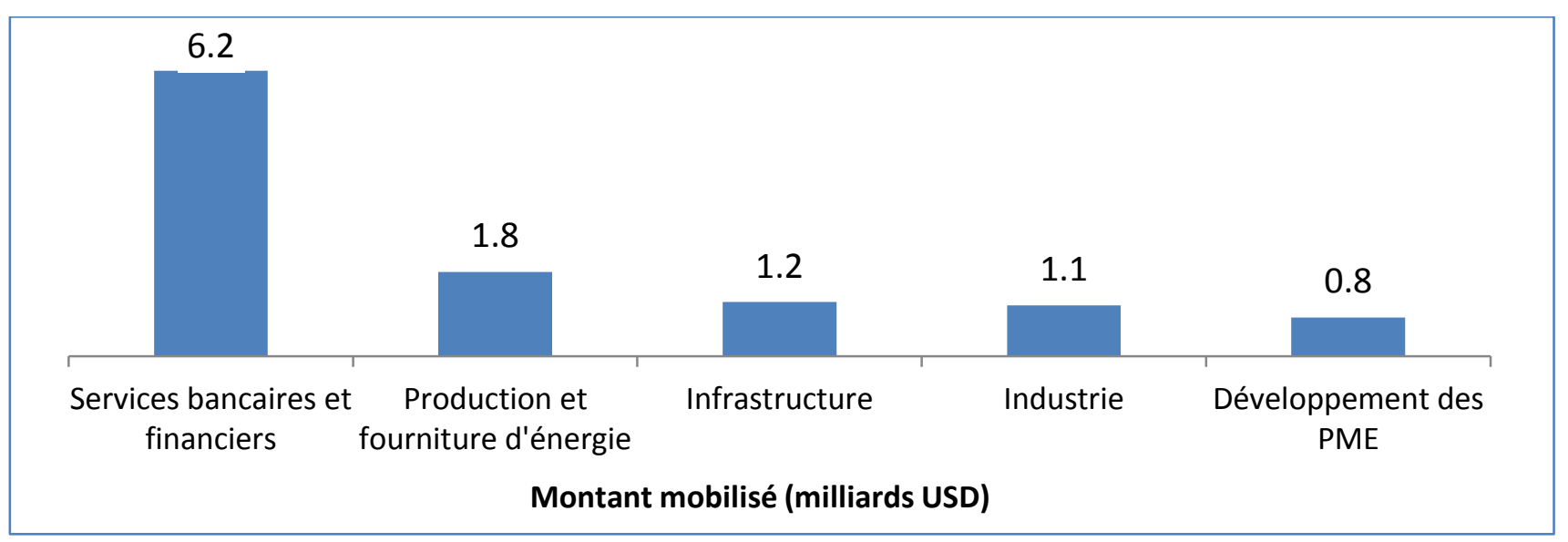

Les garanties ne sont pas couramment utilisées pour les projets liés au changement climatique.

Pour prendre en considération les discussions en cours sur les garanties dans le contexte du financement climatique, il a été demandé aux sondés d'indiquer si le projet couvert par une garantie portait sur des questions d'adaptation au changement climatique ou d'atténuation de celui-ci. Seulement 25 garanties (sur plus de 500 garanties pour lesquelles on dispose d'informations) ont été désignées comme ayant un objectif en matière de changement climatique. Ce chiffre pourrait être sous-estimé pour les raisons suivantes: i) l'enquête n'a pas donné une définition précise de ce qu'est un projet lié au changement climatique ${ }^{7}$; ii) pour certaines garanties, le descriptif du projet donne à penser que le projet aurait pu être considéré comme lié au changement climatique bien que n'ayant pas été classé comme tel, et vice versa ; et iii) plusieurs institutions ont déclaré que des garanties étaient utilisées pour des projets liés au changement climatique à partir de 2012, alors que l'enquête couvre la période 2009-2011. On pourrait donc s'attendre à ce que les garanties pour des projets liés au changement climatique soient plus répandues à l'avenir.

6. Les codes sectoriels utilisés dans cette analyse font partie des codes OCDE-CAD. Les personnes interrogées ont été encouragées à employer les codes sectoriels de l'OCDE-CAD, mais nombre d'entre elles ont utilisé les codes propres à leurs institutions. Pour procéder à l'analyse sectorielle, les informations concernant le secteur principal, le sous-secteur et le descriptif des projets ont été employées pour établir la correspondance entre les codes de chaque institution et ceux de l'OCDE-CAD.

7. Les marqueurs Rio du CAD n'ont pas été utilisés dans l'enquête afin de ne pas surcharger de travail les personnes interrogées, qui ne connaissent pas nécessairement les définitions des marqueurs. La question posée dans le cadre de l'enquête était la suivante : "Indiquez si le projet inclus l'adaptation ou attenuation du changement climatique parmi ses objectifs ». Pour que la réponse puisse être "Oui ", l'objectif d'adaptation au changement climatique ou d'atténuation de celui-ci devait être explicitement promu dans les documents du projet. Les réponses possibles étaient les suivantes : « Oui », « Non » et « Pas suivi ». 
Encadré 2 - Les garanties à court terme : les programmes de financement du commerce (PFC)

Les programmes de financement du commerce (PFC) - ou garanties à court terme - remédient à des défaillances du marché en matière de financement du commerce en accordant des garanties et des prêts aux banques afin de faciliter le commerce. Les PFC apportent aux entreprises le soutien financier dont elles ont besoin pour se livrer à des activités d'importation et d'exportation sur les marchés les plus délicats. Les différences entre les PFC et les garanties des crédits à l'exportation sont les suivantes :

- I'organisme garant a une mission de développement dans le premier cas et de facilitation des exportations dans le second ;

- les opérations ne sont pas liées dans le cas des PFC, car le garant est une IFI et non un pays spécifique ;

- la plupart des opérations couvertes par les PFC sont des exportations ou des importations entre pays en développement.

Les PFC ont mobilisé plus de 22 milliards USD de 2009 à 2011. Ces données se rapportent aux garanties à court terme accordées par la Banque européenne pour la reconstruction et le développement, la Banque interaméricaine de développement, la Banque asiatique de développement et la Société financière internationale. Les données relatives aux PFC ne sont pas pleinement comparables à celles relatives aux garanties à long terme, car une autre définition du montant mobilisé que celle proposée dans l'enquête a été utilisée.

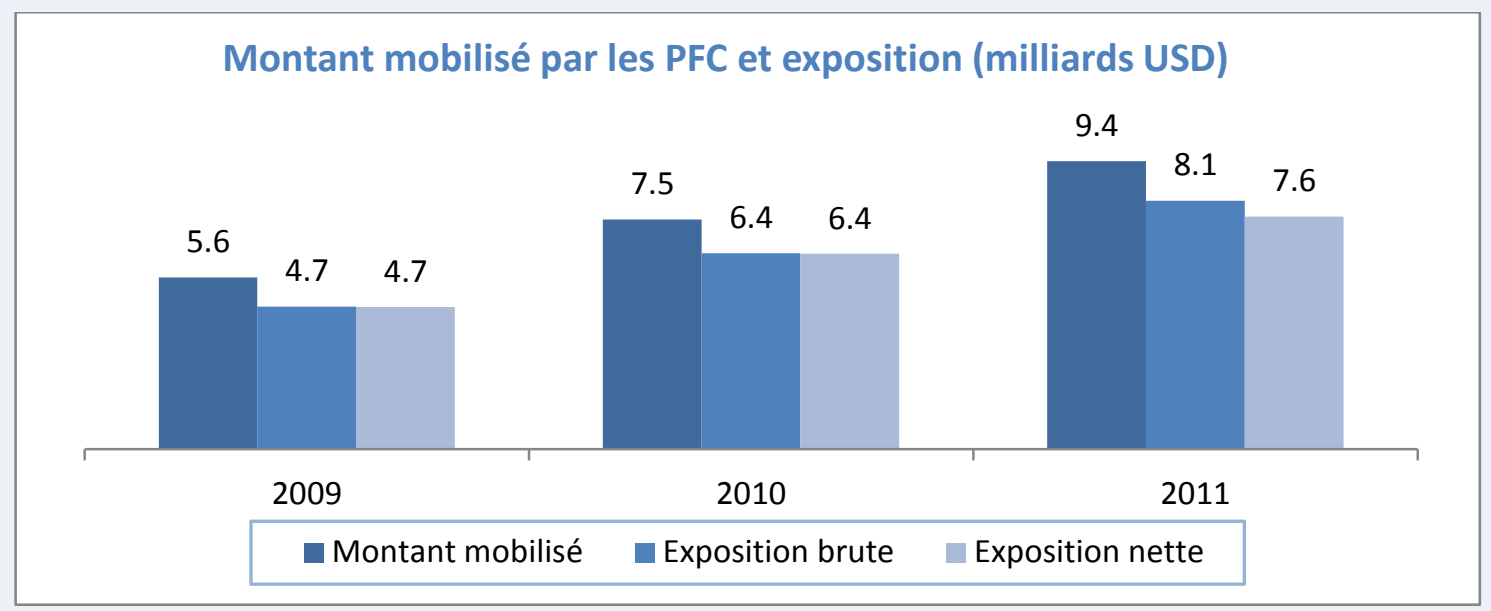




\section{II.e Quelles sont les principales caractéristiques des garanties émises à des fins de développement ?}

Les garanties couvrent les risques politiques, les risques commerciaux ou les deux. Le montant et la couverture des garanties du risque politique, principalement émises par les IFI (et en particulier par l'AMGI), excèdent largement ceux des garanties du risque commercial (tableau 1).

Tableau 1 - Garanties par type de risque couvert

\begin{tabular}{|l|c|c|c|}
\hline \multicolumn{1}{|c|}{ Risques couverts } & $\begin{array}{c}\text { Montant mobilisé } \\
\text { moyen } \\
\text { (millions USD) }\end{array}$ & $\begin{array}{c}\text { Exposition brute } \\
\text { moyenne } \\
\text { (millions USD) }\end{array}$ & $\begin{array}{c}\text { Couverture en } \\
\text { pourcentage }\end{array}$ \\
\hline Risques commerciaux et politiques & 42 & 36 & $87 \%$ \\
\hline Risques commerciaux & 4 & 2 & $44 \%$ \\
\hline Risques politiques & 18 & 18 & $99 \%$ \\
\hline
\end{tabular}

Lorsque la réassurance est employée comme outil de gestion du risque, le risque supporté par le garant est mesuré par l'exposition nette (graphique 7). Cinq institutions ont eu recours à la réassurance pour gérer le risque associé aux garanties; d'autres ont utilisé leurs ressources propres, essentiellement sous la forme de réserves de capital. Les co-garanties sont un autre mécanisme utilisé pour répartir les risques (six institutions).

Graphique 7 - Expositions brutes et nettes des garants (milliards USD)

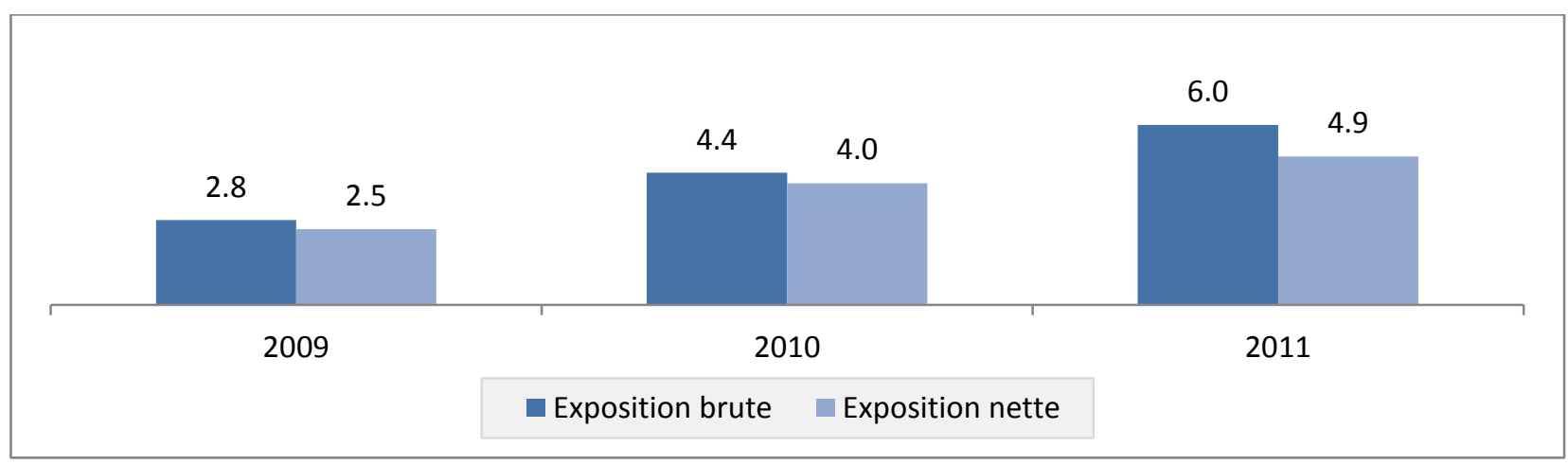

Les informations sur les commissions encaissées et les indemnisations versées sont incomplètes dans l'enquête car plusieurs organismes n'ont pas rempli les champs correspondants. D'après les données provenant de l'enquête, les recherches en ligne et les entretiens avec les organismes garants, accorder une garantie n'est généralement pas une activité déficitaire et le taux de sinistralité est très faible, de l'ordre de $1 \%$ à $2 \%$ de l'exposition du garant durant la période 2009-2011.

Les garanties sont fournies sous la forme de garanties individuelles ou de portefeuille. Les garanties individuelles sont plus répandues et couvrent, en moyenne, une plus forte proportion du risque que les garanties de portefeuille.

Les garanties de prêts sont employées plus couramment que les garanties couvrant les prises de participation, les instruments de quasi-participation et les obligations. Dans l'échantillon, plus de $70 \%$ des garanties couvrent des prêts, en termes à la fois de nombre de contrats et de montant mobilisé (graphique 8). 


\section{Graphique 8 - Instruments couverts par les garanties}
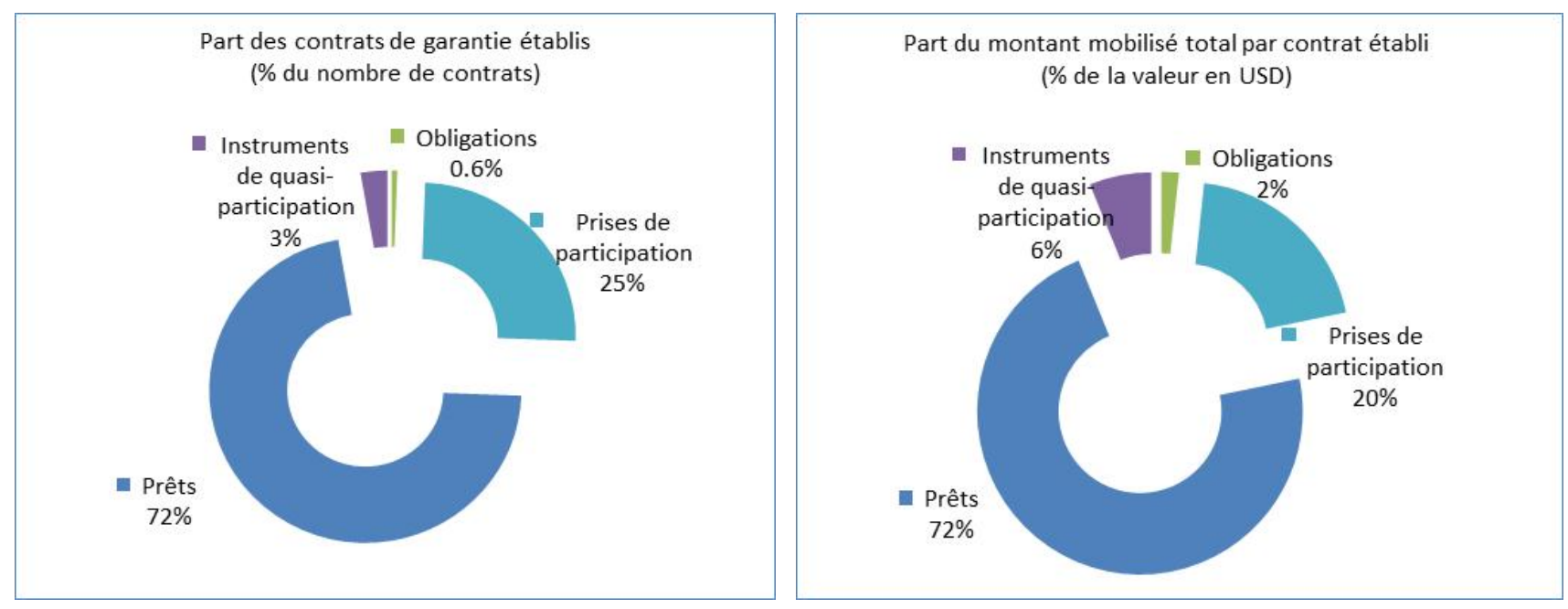

\section{Mesurer l'effet catalyseur des garanties : une première réflexion}

Les termes apports privés ayant un effet " mobilisateur ", " de levier " et "catalyseur " sont fréquemment employés au cours des discussions sur le financement du développement, mais il n'existe pour aucun d'eux une définition approuvée à l'échelle internationale. De nombreuses organisations publient des données sur les ressources qu'elles mobilisent, mais leurs méthodes de calculs varient. En effet, la signification de ces termes diffère selon les instruments auxquels ils se rapportent (par exemple, prêts, garanties) et les acteurs participant aux opérations (par exemple, organisme de développement, secteur privé).

\section{Définition du « montant mobilisé »}

Pour les besoins de l'enquête, le terme " montant mobilisé " (ou " montant obtenu par effet de levier ") ${ }^{8}$ a été défini comme suit : "Le "montant mobilisé" par une garantie est la valeur nominale totale de l'instrument (par exemple, prêt, prise de participation) auquel la garantie est attachée, quelle que soit la part de cette valeur couverte par la garantie. " Les graphiques 9 et 10 donnent des exemples destinés à éclairer le lecteur sur ce qui a motivé le choix de cette définition dans le cadre de l'enquête.

Le graphique 9 fournit l'exemple d'un projet financé par deux investisseurs, 1 et 2, dans lequel le prêt accordé par l'investisseur 1 est garanti par le garant $X$. Le « montant mobilisé » pourrait être :

1. la valeur nominale de l'instrument garanti (9 millions USD); ou

2. le coût total du projet (10 millions USD).

Choisir entre ces deux options implique d'arbitrer entre réduire au minimum le risque de double comptabilisation et éviter de sous-estimer l'effet réel de mobilisation/levier de la garantie. 
Graphique 9 - Cas où le montant garanti représente la majeure partie du coût total du projet

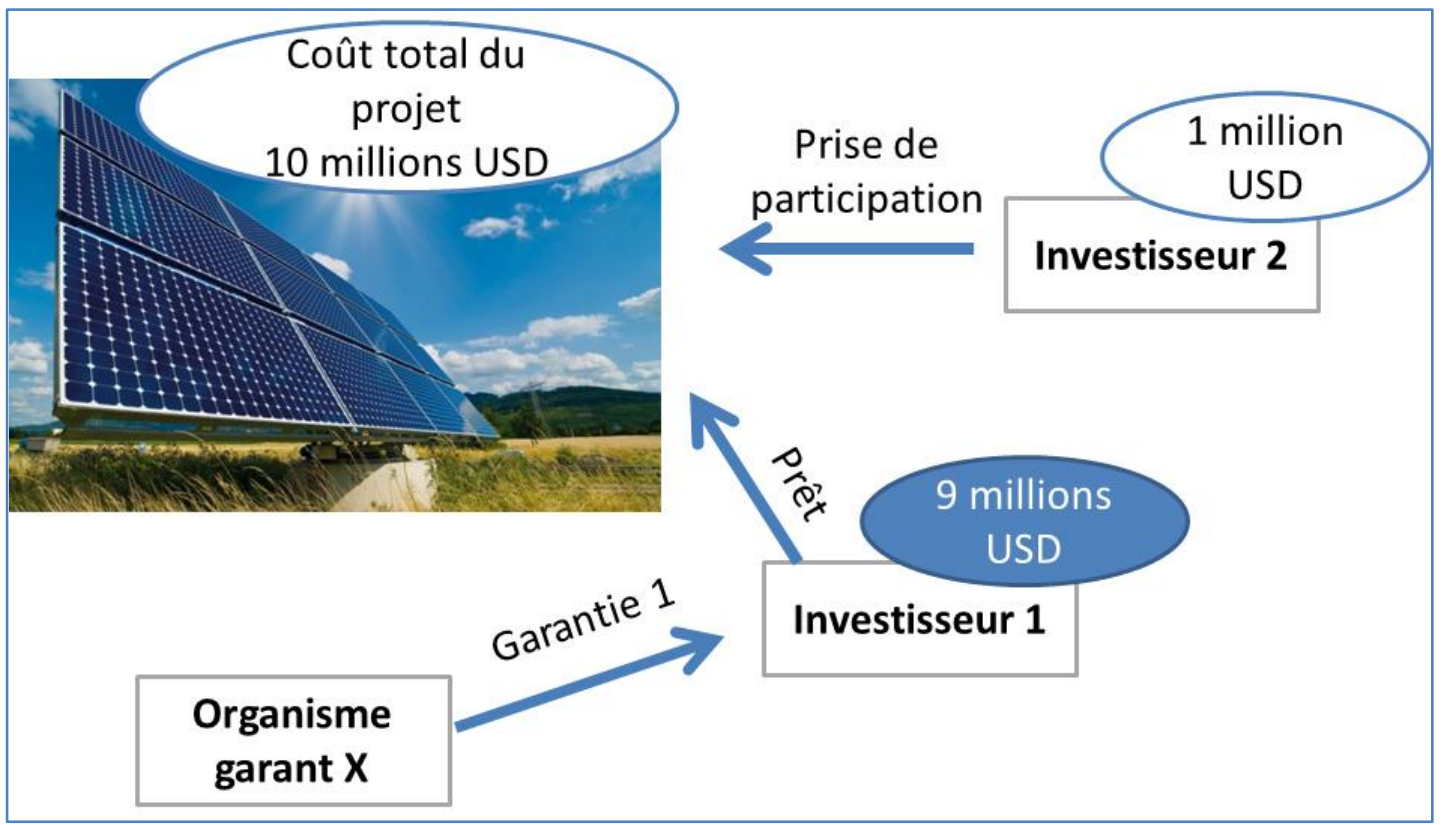

L'option(1) a été retenue pour les besoins de l'enquête. Elle repose sur l'hypothèse implicite que I'Investisseur 1 n'aurait pas investi dans le projet en l'absence de garantie, autrement dit un lien de causalité entre la garantie et l'instrument garanti est présumé.

On pourrait cependant considérer que l'option (1) est une approche " prudente ». Elle pose la question de savoir si l'Investisseur 2 aurait ou non investi dans le projet sans la garantie apportée par le Garant X, et, dans la négative, celle de savoir si le "montant mobilisé " par la garantie devrait être le coût total du projet (10 millions USD) et non le montant du prêt accordé par l'Investisseur 1 (9 millions USD).

Logiquement, plus la part de l'investissement garanti dans le coût total du projet est élevée, plus la probabilité qu'il n'aurait pas été donné suite au projet en l'absence de garantie est forte. Partant de là, on pourrait avancer que le "montant mobilisé » tel que défini dans l'enquête sous-estime de 1 million USD l'effet mobilisateur/de levier de la garantie. Cependant, si un autre garant répondant à l'enquête garantit I'Investisseur 2, il n'y a pas sous-estimation, car cette institution notifiera sa garantie pour un montant de 1 million USD, ce qui portera le montant mobilisé total à 10 millions USD.

Le graphique 10 donne un autre exemple : le prêt garanti représente une faible fraction du coût total du projet. Dans ce cas, la probabilité qu'il aurait été donné suite au projet si le prêt n'avait pas bénéficié d'une garantie est très forte, car l'essentiel des fonds est déjà disponible auprès d'autres sources (Investisseur 4). Choisir l'option (2) (le coût total du projet) pour définir le " montant mobilisé » conduirait à une surestimation de 9 millions USD, chiffre nettement supérieur à la sous-estimation résultant de l'approche prudente (1 million USD ou zéro dans le graphique 9). De surcroît, si un autre garant participait au projet et si ces deux garants notifiaient au CAD, l'option (2) porterait le montant mobilisé total à 20 millions USD (soit le double du montant effectivement mobilisé). 
Graphique 10 - Cas où le montant garanti représente une faible part du coût total du projet

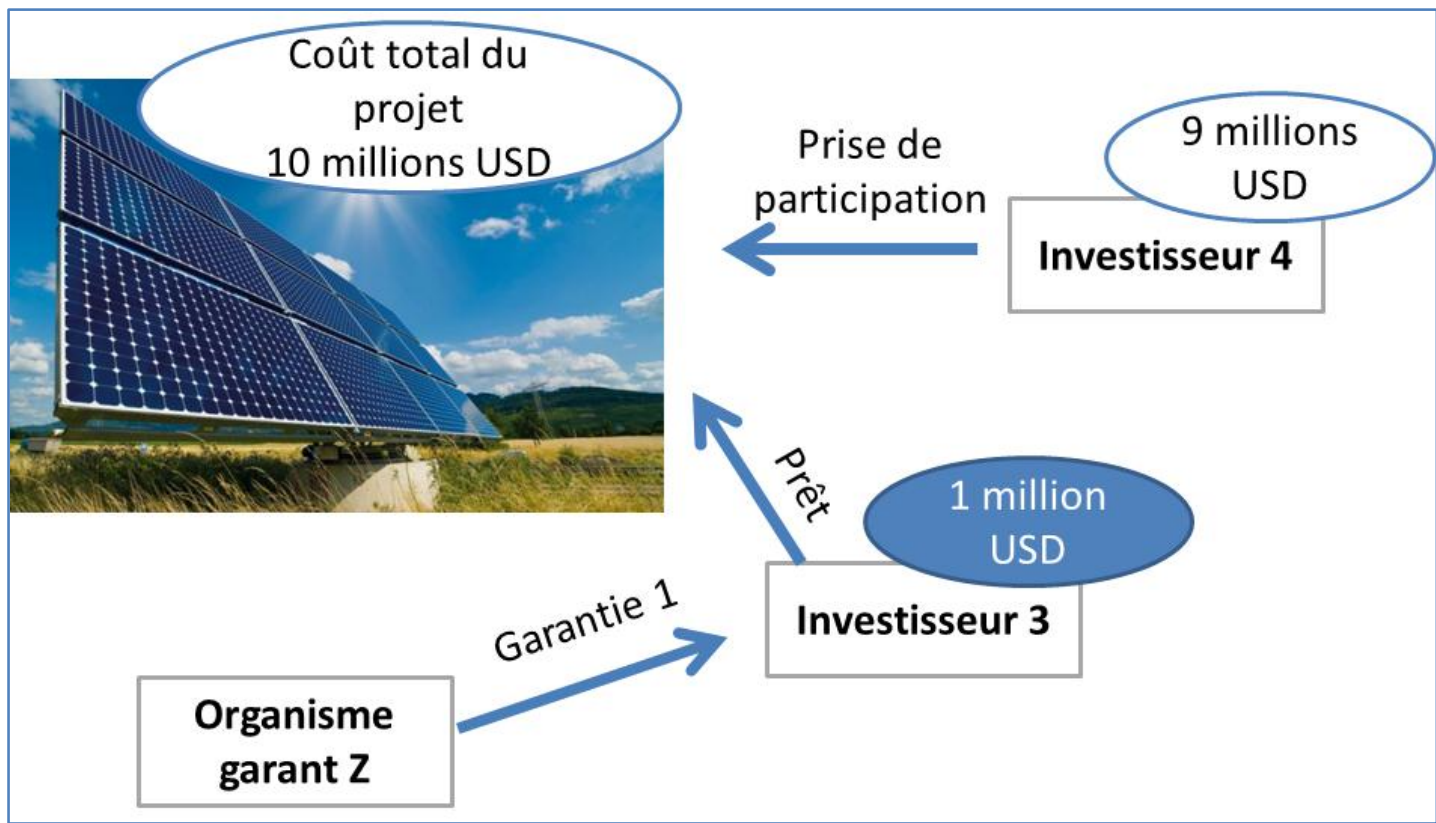

Il existe un risque de double comptabilisation en cas de co-garanties, quelle que soit la définition du montant mobilisé retenue. Le graphique 11 illustre le cas où deux garants, $X$ et $Y$, garantissent I'Investisseur 1 ; si tous deux notifient au CAD, les garanties seront déclarées deux fois. Pour éviter cet écueil, deux variables ont été incluses dans l'enquête de manière à identifier les co-garanties, et la variable " nom du garant » a permis de repérer les garanties qui ont été déclarées deux fois.

\section{Graphique 11 - Co-garanties}

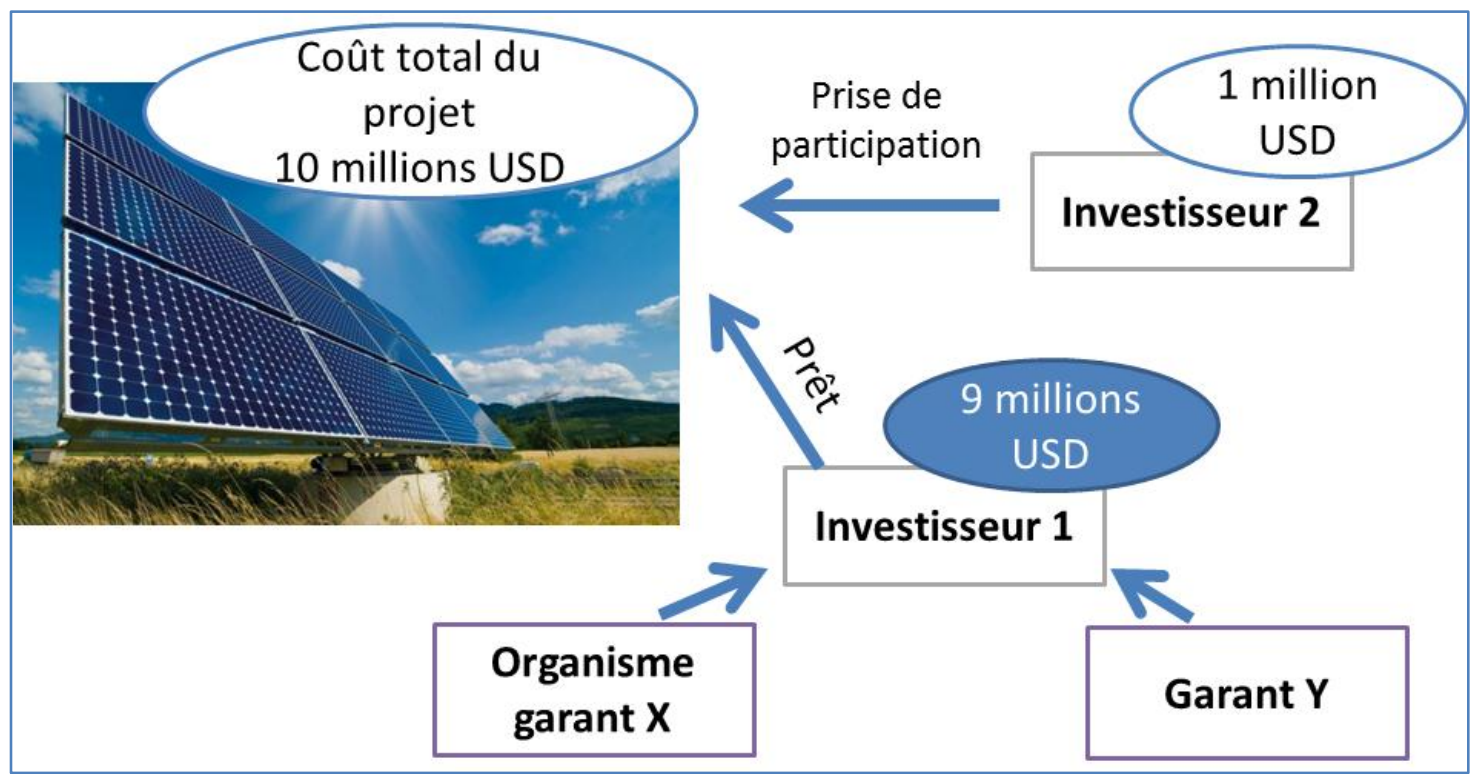




\section{III.b. Montant mobilisé vs ratio de levier - difficultés posées par le calcul de l'effet de levier}

Pour tout type de transaction, le ratio de levier mesure le volume des ressources employées par rapport au volume des ressources mobilisées. Le graphique 12 présente un exemple simple, montrant quelles sont les deux informations nécessaires au calcul: le montant mobilisé (le numérateur) et l'effort consenti par l'institution publique pour mobiliser les ressources figurant au numérateur (le dénominateur).

Graphique 12 - Ratio de levier : représentation graphique et formule (exemple)

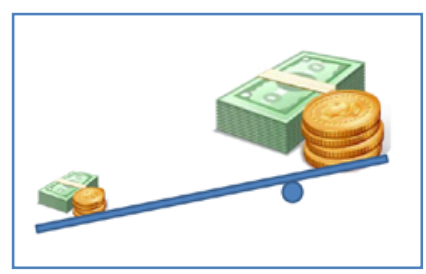

$$
\begin{aligned}
& \text { Levier }=\frac{A}{B}=\frac{\text { montant mobilisé }}{\text { effort consenti par le donneur }}=\frac{\$ 6}{\$ 1}=6 \\
& \text { Un ratio de levier de } 6 \text { pour } 1 \text { indique qu'il a fallu } 1 \text { dollar pour mobiliser } 6 \text { dollars }
\end{aligned}
$$

D'après les résultats de l'enquête, les garanties ont mobilisé plus de 15 milliards USD auprès du secteur privé à des fins de développement au cours de la période 2009-2011. Le montant mobilisé est particulièrement digne d'intérêt pour l'analyse du point de vue du bénéficiaire, car il montre l'ampleur des ressources mises à disposition des pays en développement par le jeu des garanties. Il est plus difficile de mesurer l'effort déployé par le donneur pour mobiliser ces ressources - le dénominateur de la formule du ratio de levier ${ }^{9}$.

Dans le cas d'une garantie, l'effort de l'institution publique (ou du donneur) n'est pas immédiatement visible sous la forme d'un paiement ponctuel. Cependant, il pourrait être exprimé, par exemple, en termes de risque pris par l'institution ${ }^{10}$. L'annexe 3 présente plusieurs options envisageables pour quantifier le risque pris par le garant (c'est-à-dire son exposition ex ante aux pertes éventuelles), les difficultés liées à chacune de ces options et les informations nécessaires au calcul correspondant. Elle contient aussi un exemple concret de calcul du ratio de levier effectué par la Banque mondiale.

L'analyse figurant à l'annexe 3 vise à mettre en lumière la complexité, la subjectivité et le volume des données nécessaires pour calculer le ratio de levier. II en ressort que le ratio de levier pourrait ne pas être une mesure appropriée pour quantifier l'effet catalyseur des garanties à un niveau agrégé.

Le ratio de levier peut être utilisé, comme c'est souvent le cas, au niveau des institutions pour mesurer I'utilisation stratégique des ressources, ou l'« efficience" des instruments employés pour mobiliser les ressources du secteur privé : plus le ratio de levier est élevé, plus les ressources mobilisées sont importantes par rapport aux ressources allouées par l'institution publique. Cependant, il ne faut pas perdre de vue que le ratio de levier dépend non seulement de l'effet catalyseur d'un instrument financier donné, mais aussi du contexte et des conditions de marché dans lesquels cet instrument est employé. Un instrument aura probablement un ratio de levier plus faible lorsqu'il sera utilisé dans un PMA que lorsqu'il le sera dans un PRI (car le secteur public devra vraisemblablement déployer davantage d'efforts dans le cas d'un PMA que dans celui d'un PRI pour rendre les investissements dans ces pays attrayants pour le secteur privé).

9. En particulier pour les garanties. Dans le cas d'un « instrument d'apport ", l'effort du donneur correspond au montant du prêt, lequel est facilement quantifiable. Dans le cas d'un prêt, les questions d'attribution/de causalité rendent plus difficile la quantification du numérateur - du montant mobilisé.

10

On peut citer comme autres options envisageables les indemnités effectivement versées en cas de défaillance, les souscriptions au capital de l'organisme émettant les garanties ou la différence entre la commission de garantie facturée par l'institution publique et la commission de garantie au taux du marché. 
Dès lors que la maximisation du ratio de levier est considérée comme mesurant l'« efficience " des institutions, il est à craindre que les ressources aillent principalement aux PRI, car c'est dans ces pays que le contexte permettrait cette maximisation. Les ressources publiques seront plus "efficientes" au sens où il faut moins de ressources publiques pour mobiliser les ressources du secteur privé tout en étant moins " efficaces \#, car ces ressources ne seront pas investies dans les pays qui ont le plus besoin de ressources extérieures pour financer leur développement, à moins que les ressources publiques libérées par l'accroissement des investissements du secteur privé dans les PRI ne soient réaffectées aux PMA.

Un autre point à prendre en considération lorsque l'on utilise le ratio de levier comme indicateur de l'efficience est que, en termes d'attribution du montant mobilisé, plus le ratio de levier est élevé, moins les ressources mobilisées sont susceptibles d'être attribuables à l'intervention des pouvoirs publics.

\section{Défis à relever et prochaines étapes}

En s'appuyant sur les résultats de l'enquête, le CAD étudiera une proposition de collecte périodique des données sur les garanties devant permettre de quantifier, au niveau international, les ressources mises à disposition (montant mobilisé) des/dans les pays en développement par le biais des mécanismes de garantie et de valoriser ainsi l'utilisation de ces instruments par les donneurs.

\section{Champ des futures analyses sur les garanties: motivation développementale vs impact sur le développement}

Les résultats de l'enquête ont montré que la frontière entre les garanties pour le développement (telles que définies dans le questionnaire) et les garanties accordées pour raison commerciale n'est pas clairement définie. Le développement et le commerce peuvent même être deux motifs interdépendants, par exemple lorsque la viabilité commerciale est indispensable pour qu'un organisme de développement financé sur fonds public garantisse un projet ${ }^{11}$.

La meilleure solution consisterait donc peut-être à définir le champ de la collecte des données en fonction du cadre institutionnel. Les organismes publics qui émettent des garanties sont les organismes d'aide, les IFD, les IFI et les organismes de crédit à l'exportation. Les données sur les garanties des crédits à l'exportation sont déjà collectées au niveau des activités par le Groupe de l'OCDE sur les crédits à l'exportation, et des travaux sont en cours en vue d'harmoniser cette collecte avec le système du CAD. Par conséquent, seules les garanties émises par les organismes d'aide, les IFD et les IFI feraient l'objet d'une collecte de données par le CAD. Les études analytiques sur les dispositifs de garantie pourraient porter à la fois sur les garanties motivées par des considérations de développement et sur celles motivées par des considérations commerciales, si l'analyste le juge utile.

\section{Questions de confidentialité}

Pour cette enquête, la plupart des pays ont été en mesure de fournir des informations détaillées au niveau des garanties individuelles. Cela a permis de calculer les agrégats par pays bénéficiaire, secteur, etc. Cependant, les informations relatives aux garanties individuelles sont généralement classées comme étant des informations commerciales confidentielles, et les réponses données dans le cadre de l'enquête par les différents pays et institutions ne peuvent être publiées ou communiquées. Lorsqu'il étudiera la possibilité d'une collecte périodique des données, le CAD souhaiterait également examiner le champ d'application de la collecte des données qui seraient destinées à publication.

\footnotetext{
${ }^{11}$ Exercer une activité à caractère commercial est un critère d'éligibilité d'un projet pour la plupart des financements et garanties des IFD et IFI.
} 
En outre, et également pour des raisons de confidentialité, un certain nombre d'IFI n'ont pas été en mesure de fournir des informations au niveau des garanties individuelles, principalement dans le cas de garanties à court terme. Une solution consisterait en l'occurrence à collecter les données sur les garanties individuelles pour les contrats à long terme et à ne collecter que les agrégats par pays bénéficiaire et par secteur pour les garanties à court terme.

\section{Méthodologie employée pour calculer le montant mobilisé par les garanties}

Les données relatives au montant mobilisé, tel que défini dans l'enquête, ne sont pas toujours directement disponibles, et la méthodologie employée pour calculer ce montant varie d'une institution à l'autre. Pour développer la collecte de statistiques sur les garanties, il est essentiel de disposer d'une définition reconnue afin d'assurer la cohérence et la comparabilité des données.

La définition du " montant mobilisé » utilisée dans l'enquête a été jugée appropriée pour une future collecte de données. Toutefois, il a en outre été suggéré de : 1 ) rendre compte du coût total du projet, pour s'assurer qu'une approche prudente n'aboutit pas à sous-estimer l'effet catalyseur des garanties ; 2) estimer le montant mobilisé au moyen de l'exposition nette, car cette dernière représente le risque supporté par le garant (et correspond au passif éventuel dans le bilan du garant) et réduit le risque de double comptabilisation.

\section{$\underline{\text { Ratio de levier }}$}

L'enquête permet de penser que le ratio de levier n'est pas un moyen approprié de quantifier l'effet catalyseur des garanties à un niveau agrégé. Dans la pratique, collecter le volume de données nécessaires pour le calculer ne se révèlerait pas d'un bon rapport coût-efficacité. De surcroît, les comparaisons entre pays risquent d'être biaisées s'il n'est pas bien rendu compte de la probabilité de défaillance - indicateur complexe à mesurer.

La mesure de l'effort des donneurs au niveau des garanties individuelles n'a pas été traitée spécifiquement dans l'enquête, car cette question fait partie du débat plus large qui se déroule actuellement dans le cadre du financement du développement de l'après 2015. La prochaine proposition visera donc seulement à rendre compte de l'effet catalyseur des garanties du point de vue du bénéficiaire (montant mobilisé). Une mesure de l'effort des donneurs serait prématurée à ce stade, mais pourrait être élaborée en temps opportun. 


\section{ANNEXE 1 - ÉCHANTILLON ET TAUX DE RÉPONSE}

L'enquête a été organisée par l'intermédiaire du Groupe de travail du CAD sur les statistiques sur le financement du développement (GT-STAT) en collaboration avec les correspondants statistiques dans les pays membres et non membres du CAD et dans les IFI.

L'échantillon comprend des IFD, des organismes d'aide et des ministères des affaires étrangères de 24 donneurs membres du CAD ${ }^{12}$ ainsi que 17 IFI. La liste des pays et institutions de l'échantillon figure dans le tableau A2. Les organismes de crédit à l'exportation n'ont pas été inclus dans l'échantillon parce que le développement n'est pas leur objectif principal.

Les informations ont été collectées au niveau des garanties individuelles en ce qui concerne les garanties à long terme ${ }^{13}$ émises entre 2009 et 2011. Les données relatives aux garanties à court terme ont été recueillies à un niveau agrégé pour des raisons de confidentialité, également pour la période 2009-2011.

Le taux de réponse à l'enquête est de $85 \%, 20$ pays (institutions bilatérales) et $15 \mathrm{IFI}$ ayant répondu à l'enquête. Globalement, 17 pays ou institutions ont accordé des garanties pour le développement et 4 autres institutions prévoient de recourir à ces instruments à des fins de développement dans un futur proche (tableau A1).

Tableau A1 - Réponses à l'enquête, par type d'institution - garanties à court terme et à long terme

\begin{tabular}{|c|c|c|c|}
\hline & Institutions bilatérales & Institutions multilatérales & Total \\
\hline Accordant des garanties & 8 & 9 & 17 \\
\hline N'accordant pas de garanties & 10 & 4 & 14 \\
\hline $\begin{array}{l}\text { Prévoyant d'accorder des } \\
\text { garanties dans un futur } \\
\text { proche }\end{array}$ & 2 & 2 & 4 \\
\hline Total & 20 & 15 & 35 \\
\hline
\end{tabular}

De 2009 à 2011, 1170 garanties à long terme et des milliers de garanties à court terme ${ }^{14}$ ont été émises. Cinquante-cinq garanties ont été exclues de l'échantillon; les raisons de leur exclusion sont indiquées dans l'encadré 3.

L'analyse des données dans la section II porte essentiellement sur les garanties qui ont mobilisé des fonds du secteur privé. Ces garanties représentent plus de $90 \%$ du montant mobilisé dans l'échantillon. Les garanties intra-organismes n'entraient pas dans le champ de l'enquête, ce qui pourrait expliquer le faible pourcentage

\footnotetext{
12. Douze donneurs non membres du CAD ont également été invités à participer à l'enquête. Quatre d'entre eux ont répondu ; aucun d'eux n'accorde de garantie pour le développement. Ces pays n'ont pas été inclus dans l'analyse présentée dans ce rapport.

13. Les garanties à long terme couvrent les risques pendant un an ou plus.

14. On ne dispose pas du nombre exact de contrats de garantie à court terme émis.
} 
obtenu pour le financement public. De plus, les bénéficiaires des garanties ${ }^{15}$ sont principalement des sociétés privées (plus de $80 \%$ ) ; les bénéficiaires publics et privés ont été inclus dans l'analyse.

\section{Encadré 3 - CRITÈRES DE NETTOYAGE DES DONNÉES}

Au total, 55 transactions déclarées ont été exclues de l'analyse pour les raisons suivantes (quelques garanties ont été exclues pour plus d'une raison):

- L'investisseur n'était pas de type privé. Comme l'enquête a pour principal objet d'estimer le montant mobilisé auprès du secteur privé, les garanties pour lesquelles l'investisseur est un organisme public ont été exclues de l'analyse (16 garanties).

- Le pays bénéficiaire n'était pas un pays éligible à l'APD (36 garanties).

- L'instrument garanti était lui-même une garantie (2 garanties).

- Manque de données sur le montant mobilisé et sur l'exposition (2 garanties).

- Utilisation de co-garanties. Dans le cas où deux institutions de l'échantillon sont co-garantes du même instrument sous-jacent, la même garantie est déclarée deux fois. Pour éviter une double comptabilisation, une seule des deux garanties a été incluse dans l'analyse ( 3 garanties).

15. Le bénéficiaire est l'entité située dans le pays en développement où l'investissement est réalisé in fine. Dans le cas d'un prêt, c'est

l'emprunteur dont la dette sera payée par le garant si le débiteur ne s'acquitte pas de sa dette auprès de l'investisseur. 
Tableau A2 - Liste des pays et institutions composant l'échantillon

\begin{tabular}{|c|c|c|}
\hline Pays et institutions interrogés & $\begin{array}{l}\text { Garanties pour le } \\
\text { développement ? }\end{array}$ & $\begin{array}{c}\text { Montant mobilisé } \\
\text { 2009-2011 } \\
\text { (millions USD) }\end{array}$ \\
\hline Australie - AUSAID & Pas de réponse & \\
\hline Autriche - Oesterreichische Entwicklungsbank AG & Oui & 304.3 \\
\hline Belgique - DG Développement, ministère des Affaires étrangères & Non & \\
\hline Canada - Exportation et développement Canada & Non & \\
\hline Danemark - DANIDA & Non & \\
\hline Union Européenne - EuropeAid ; BEI & Pas de réponse & \\
\hline Finlande - FINNVERA & Oui & 209.1 \\
\hline France - Agence française de développement ; PROPARCO & Oui & 1116.1 \\
\hline Allemagne - KfW; DEG & Oui & 62.9 \\
\hline Grèce - Ministère des Affaires Étrangères & Non & \\
\hline Irlande - DFA & Pas de réponse & \\
\hline $\begin{array}{l}\text { Italie - Ministère des Affaires Étrangères - Direction générale de la coopération pour le } \\
\text { développement }\end{array}$ & Dans un futur proche & \\
\hline Japon - MFA; JICA; JBIC & Non & \\
\hline Corée - KEXIM & Dans un futur proche & \\
\hline $\begin{array}{l}\text { Luxembourg - Direction de la coopération pour le développement, ministère des Affaires } \\
\text { étrangères }\end{array}$ & Non & \\
\hline $\begin{array}{l}\text { Pays-Bas - Atradius Dutch State Business, ministère de la Coopération pour le } \\
\text { développement, DG Commerce international, Économie extérieure }\end{array}$ & Non & \\
\hline Nouvelle Zélande - NZAID & Pas de réponse & \\
\hline Norvège - NORAD ; NORFUND & Oui & 29.7 \\
\hline Portugal - SOFID & Oui & 3.7 \\
\hline Espagne - CESCE & Non & \\
\hline Suède - SIDA & Oui & 12.6 \\
\hline Suisse - SECO & Non & \\
\hline Royaume-Uni - DFID ; UK Export Finance & Non & \\
\hline États-Unis - USAID ; OPIC & Oui & 5621.2 \\
\hline Fonds arabe pour le développement économique et social (Fonds arabe) & Non & \\
\hline Banque arabe pour le développement économique en Afrique (BADEA) & Non & \\
\hline Banque de développement des Caraïbes (BDC) & Non & \\
\hline Credit Guarantee and Investment Facility (CGIF) & Dans un futur proche & \\
\hline Fonds d'investissement pour le climat (FIC) & Pas de réponse & \\
\hline Banque européenne pour la reconstruction et le développement (BERD) & Oui*** & \\
\hline Banque interaméricaine de développement (BID) & Oui**** & 69.8 \\
\hline $\begin{array}{l}\text { Banque internationale pour la reconstruction et le développement/International } \\
\text { Development Association (BIRD/IDA) }\end{array}$ & Oui & 1496.2 \\
\hline Fonds international de développement agricole (FIDA) & Non & \\
\hline Société financière internationale (SFI) & Oui**** & 1199.9 \\
\hline Banque islamique de développement [BisD (ICIEC)] & Oui & 430.9 \\
\hline Agence multilatérale de garantie des investissements (AMGI) & Oui & 4467.9 \\
\hline Nordic Development Fund (NDF) & Dans un futur proche & \\
\hline Fonds de I'OPEP pour le développement international & Pas de réponse & \\
\hline Private Infrastructure Development Group (PIDG) - GuarantCo & Oui $^{\mathrm{a}}$ & 144.2 \\
\hline \multicolumn{3}{|c|}{$\begin{array}{l}\text { * Accorde des garanties à court terme et à long terme. Seules les garanties à court terme ont été notifiées, car aucune garantie à long terme n'a été émise au cours de la } \\
\text { période 2009-2011. Les données relatives aux garanties à court terme ne sont pas présentées dans ce tableau, car elles ne sont pas comparables aux données relatives aux } \\
\text { garanties à long terme. } \\
\text { ** Accorde des garanties à court terme et à long terme. Seules les garanties à long terme ont été notifiées, car aucune garantie à court terme n’a été émise au cours de la } \\
\text { période 2009-2011. }\end{array}$} \\
\hline \multicolumn{3}{|c|}{$\begin{array}{l}\text { *** Accorde des garanties à court terme et à long terme. Ces deux types de garanties ont été notifiés dans le cadre de l'enquête, cependant les garanties à long terme ont été } \\
\text { exclues de l'analyse car l'investisseur était une organisation multilatérale (non privée). }\end{array}$} \\
\hline
\end{tabular}




\section{ANNEXE 2 - PAYS BÉNÉFICIANT DE GARANTIES À LONG TERME}

\begin{tabular}{|c|c|c|}
\hline Région & Pays bénéficiaire & $\begin{array}{c}\text { Montant mobilisé, } 2009 \\
2011 \\
\text { (millions USD) }\end{array}$ \\
\hline \multirow{45}{*}{ Afrique } & Afrique du Sud & 342.5 \\
\hline & Algérie & 173.2 \\
\hline & Angola & 13.6 \\
\hline & Bénin & 15.2 \\
\hline & Botswana & 825.3 \\
\hline & Burkina Faso & 84.6 \\
\hline & Burundi & 4.1 \\
\hline & Cameroun & 176.4 \\
\hline & Centrafricaine, Rép. & 0.8 \\
\hline & Congo, Rép. & 19.9 \\
\hline & Congo, Rép. dém. & 25.4 \\
\hline & \begin{tabular}{|l} 
Côte d'Ivoire \\
\end{tabular} & 219.8 \\
\hline & Djibouti & 25.4 \\
\hline & \begin{tabular}{|l|} 
Egypte \\
\end{tabular} & 72.5 \\
\hline & Ethiopie & 224.0 \\
\hline & Gabon & 43.8 \\
\hline & Ghana & 729.9 \\
\hline & Guinée & 12.3 \\
\hline & Kenya & 472.7 \\
\hline & \begin{tabular}{|l} 
Liberia \\
\end{tabular} & 180.2 \\
\hline & Libye & 7.4 \\
\hline & Madagascar & 140.8 \\
\hline & Malawi & 6.0 \\
\hline & Mali & 47.6 \\
\hline & Maroc & 0.2 \\
\hline & Mauritanie & 5.3 \\
\hline & Mauritius & 53.6 \\
\hline & Mozambique & 36.0 \\
\hline & Namibie & 74.8 \\
\hline & Niger & 4.9 \\
\hline & Nigeria & 368.9 \\
\hline & \begin{tabular}{|l} 
Ouganda \\
\end{tabular} & 42.8 \\
\hline & Rwanda & 82.3 \\
\hline & Sénégal & 378.8 \\
\hline & Seychelles & 135.5 \\
\hline & Sierra Leone & 12.7 \\
\hline & Somalie & 0.1 \\
\hline & Swaziland & 1.2 \\
\hline & \begin{tabular}{|l} 
Tanzanie \\
\end{tabular} & 28.2 \\
\hline & Tchad & 19.5 \\
\hline & Togo & 54.9 \\
\hline & Tunisie & 325.7 \\
\hline & Zambie & 28.0 \\
\hline & Zimbabwe & 40.0 \\
\hline & Pays non spécifié & 525.6 \\
\hline & Sous-total: Afrique & 6082.1 \\
\hline
\end{tabular}

\begin{tabular}{|c|c|c|}
\hline Région & Pays bénéficiaire & $\begin{array}{l}\text { Montant mobilisé, } \\
\text { 2009-2011 } \\
\text { (millions USD) }\end{array}$ \\
\hline \multirow{21}{*}{ Amérique } & Antigua et Barbuda & 0.05 \\
\hline & Argentine & 4.0 \\
\hline & Bolivie & 12.2 \\
\hline & Brésil & 127.1 \\
\hline & Chili & 30.0 \\
\hline & Colombie & 89.2 \\
\hline & Costa Rica & 78.0 \\
\hline & \begin{tabular}{|l} 
Dominicaine, Rép. \\
\end{tabular} & 8.7 \\
\hline & Dominique & 0.0 \\
\hline & El Salvador & 34.0 \\
\hline & Equateur & 15.2 \\
\hline & Guatemala & 54.3 \\
\hline & Haïti & 63.3 \\
\hline & \begin{tabular}{|l|} 
Honduras \\
\end{tabular} & 158.9 \\
\hline & Mexique & 386.6 \\
\hline & Nicaragua & 41.3 \\
\hline & Panama & 197.4 \\
\hline & Paraguay & 85.2 \\
\hline & Pérou & 234.2 \\
\hline & \begin{tabular}{|l|} 
Uruguay \\
\end{tabular} & 0.1 \\
\hline & Pays non spécifié & 246.7 \\
\hline & Sous-total: Amérique & 1866.3 \\
\hline \multirow{28}{*}{ Asie } & Afghanistan & 107.3 \\
\hline & Arménie & 23.0 \\
\hline & Azerbaïdjan & 67.5 \\
\hline & Bangladesh & 9.1 \\
\hline & Cambodge & 17.1 \\
\hline & Chine & 254.7 \\
\hline & Cisjordanie et bande de Gaza & 102.6 \\
\hline & Géorgie & 104.3 \\
\hline & Inde & 392.5 \\
\hline & Indonésie & 762.3 \\
\hline & Irak & 32.3 \\
\hline & Jordanie & 154.7 \\
\hline & Kazakhstan & 381.9 \\
\hline & Kirghize, Rép. & 8.0 \\
\hline & Liban & 90.9 \\
\hline & Mongolie & 45.3 \\
\hline & Népal & 0.3 \\
\hline & Ouzbékistan & 0.8 \\
\hline & Pakistan & 169.5 \\
\hline & Philippines & 48.0 \\
\hline & Sri Lanka & 26.7 \\
\hline & Syrie & 9.3 \\
\hline & Tadjikistan & 4.8 \\
\hline & Thaïlande & 306.1 \\
\hline & Timor-Leste & 0.2 \\
\hline & \begin{tabular}{|l|} 
Vietnam \\
\end{tabular} & 102.4 \\
\hline & Pays non spécifié & 326.3 \\
\hline & Sous-total: Asie & 3547.8 \\
\hline \multirow{9}{*}{ Europe } & Albanie & 117.3 \\
\hline & Bosnie-Herzégovine & 243.4 \\
\hline & Kosovo & 74.0 \\
\hline & Macédoine, ERY & 202.3 \\
\hline & Moldova & 38.7 \\
\hline & Serbie & 1172.4 \\
\hline & Turquie & 1053.3 \\
\hline & Ukraine & 222.4 \\
\hline & Pays non spécifié & 149.8 \\
\hline & Sous-total: Europe & 3273.5 \\
\hline & Multiples régions & 527.8 \\
\hline & Total & 15297.6 \\
\hline
\end{tabular}




\section{ANNEXE 3 - CALCULER LE RATIO DE LEVIER : EXEMPLE CONCRET, DONNÉES REQUISES ET MÉTHODOLOGIES POSSIBLES}

Le graphique $A 3$ donne un exemple de co-garantie. Une banque commerciale établie en Chine prête 825 millions USD à une société d'énergie. La durée du prêt est de 20 ans. Sinosure - l'agence chinoise de crédit à l'exportation - garantit les 15 premières années du projet; la Banque mondiale garantit les 5 dernières années.

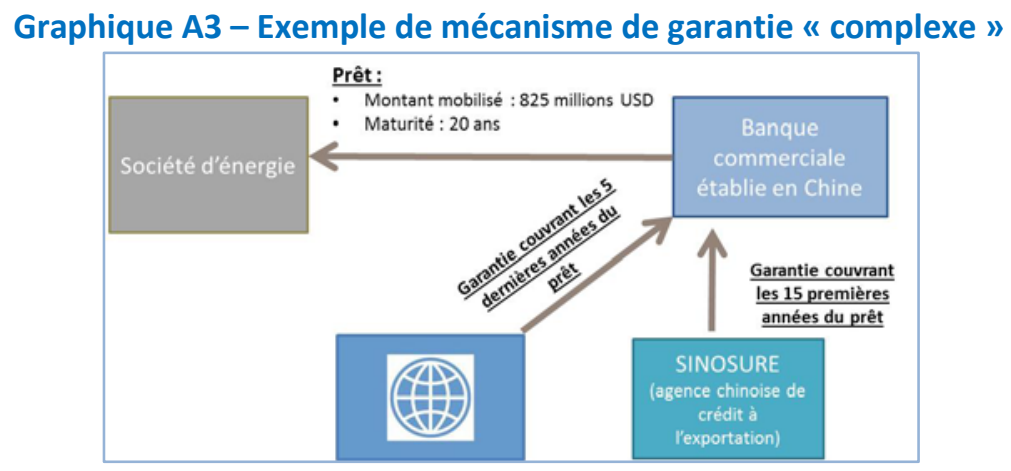

La Banque mondiale a calculé l'effet de levier produit par la garantie qu'elle apporte comme étant le rapport entre la valeur nominale du prêt garanti et la valeur actuelle de l'exposition de la Banque mondiale ${ }^{16}$. Le montant mobilisé est de 825 millions USD. L'exposition est la somme maximale que la Banque mondiale devra verser dans le cas où la société ne rembourse pas la banque commerciale au cours des cinq dernières années du prêt. Cette somme, le montant résiduel du prêt, s'élève à 242 millions USD (graphique A4). La valeur actuelle de l'exposition est de 121 millions USD, d'où un ratio de levier de 7 pour 1.

Graphique A4 - Amortissement du prêt

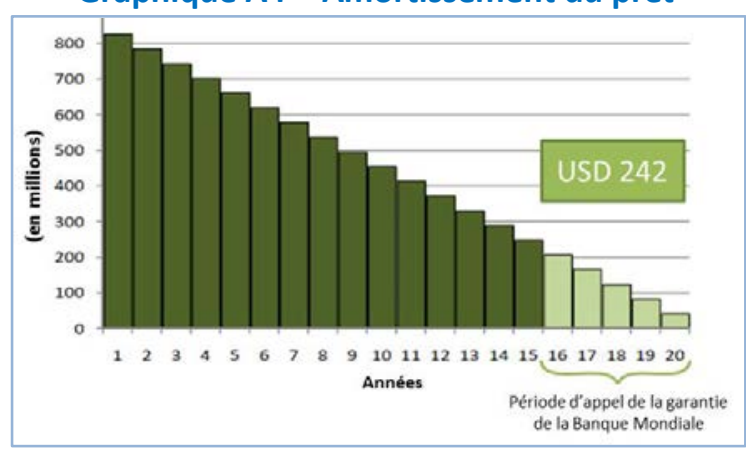

Ce n'est qu'une des nombreuses façons de calculer le ratio de levier. Le tableau A5 illustre d'autres possibilités de calculer le ratio de levier, en particulier de quantifier son dénominateur. Pour simplifier, le numérateur c'est-à-dire le montant mobilisé - est défini comme dans l'enquête.

Une importante limite de la plupart des options proposées est que la probabilité de défaillance n'entre pas en ligne de compte dans le calcul. Le risque pris par le garant se compose de deux éléments : l'exposition nette (qui est la somme maximale que le garant devra payer en cas de défaillance) et la probabilité de défaillance, laquelle varie selon le contexte. Le fait que deux garants ont la même exposition nette (par exemple,

16. Source : http://siteresources.worldbank.org/INTGUARANTEES/Resources/BotswanapowerMorupule.pdf 
10 millions USD) ne signifie pas qu'ils prennent chacun le même risque (ou font chacun le même effort) ${ }^{17}$. Par conséquent, si la probabilité de défaillance n'est pas prise en compte, les comparaisons entre les différents garants risquent d'être biaisées.

D'autres variables à prendre en considération sont le type de risque et la devise dans laquelle l'instrument garanti est libellé. Une garantie qui couvre à la fois les risques commerciaux et les risques politiques a une plus forte probabilité d'être appelée qu'une garantie ne couvrant que l'un ou l'autre de ces risques. Une garantie couvrant un prêt libellé dans la monnaie locale d'un pays en développement implique, au moins en théorie, un risque plus élevé qu'une garantie couvrant un prêt libellé dans une devise forte. Ne pas prendre ces caractéristiques en compte est une autre source de biais lorsque l'on compare des garants différents.

Les données requises pour calculer le ratio de levier varient en fonction de la méthodologie employée et de chaque projet. Dans la pratique, il n'y a pas de méthodologie universelle et, au niveau agrégé, il semble irréaliste de vouloir collecter le volume d'informations nécessaires pour calculer le ratio.

Tableau A5 - Options envisageables pour calculer le ratio de levier des garanties

\begin{tabular}{|c|c|c|}
\hline $\begin{array}{l}\text { Options pour le } \\
\text { dénominateur }\end{array}$ & Difficultés & Informations nécessaires au calcul \\
\hline $\begin{array}{l}\text { 1. Exposition } \\
\text { nette }\end{array}$ & $\begin{array}{l}\text { La probabilité que le risque se matérialise est faible. En } \\
\text { conséquence, l'effort de l'institution publique est } \\
\text { surestimé et l'effet de levier est sous-estimé. En outre, } \\
\text { comme les caractéristiques de la garantie et du contexte } \\
\text { ne sont pas prises en compte, les comparaisons entre les } \\
\text { garants risquent d'être biaisées. }\end{array}$ & $\begin{array}{l}\text { - Montant mobilisé } \\
\text { - } \text { Exposition nette } \\
\text { - } \text { Durée du prêt** } \\
\text { - Période durant laquelle la garantie peut être } \\
\text { - } \text { appelée } \\
\text { - Taux d'actualisation pour calculer la valeur } \\
\text { actuelle*** }\end{array}$ \\
\hline $\begin{array}{l}\text { 2. Exposition } \\
\text { nette } X \\
\text { probabilité de } \\
\text { défaillance }\end{array}$ & $\begin{array}{l}\text { Dans la pratique, la probabilité de défaillance est propre } \\
\text { au projet et c'est un indicateur complexe à mesurer, } \\
\text { même au niveau du projet. S'il n'en est pas rendu } \\
\text { compte correctement, les comparaisons entre les } \\
\text { garants risquent d'être biaisées. }\end{array}$ & $\begin{array}{l}\text { - Montant mobilisé } \\
\text { - Exposition nette } \\
\text { - } \text { Durée du prêt } \\
\text { - Période durant laquelle la garantie peut être } \\
\text { - } \text { appelée } \\
\text { - Taux d'actualisation pour calculer la valeur } \\
\text { - } \text { actuelle } \\
\text { - Prime de réassurance }\end{array}$ \\
\hline $\begin{array}{l}\text { 3. Montant du } \\
\text { capital } \\
\text { immobilisé } \\
\text { (provision) }\end{array}$ & $\begin{array}{l}\text { Les organismes provisionnent une certaine somme par } \\
\text { dollar garanti pour constituer une " réserve » en cas de } \\
\text { défaillance (par ex., } 25 \text { cents par dollar garanti). Cette } \\
\text { option tient compte du fait que l'organisme ne peut } \\
\text { utiliser la somme immobilisée pour investir dans } \\
\text { d'autres projets. Cependant, le montant du capital } \\
\text { immobilisé ne constitue pas un apport et sera disponible } \\
\text { dans le portefeuille de l'institution après expiration de la } \\
\text { garantie (sauf en cas de défaillance). Par conséquent, } \\
\text { cette option surestime l'effort de l'institution publique } \\
\text { et sous-estime l'effet de levier des garanties. }\end{array}$ & $\begin{array}{l}\text { - Montant mobilisé } \\
\text { - Provision (montant des ressources } \\
\text { immobilisées) } \\
\text { - Durée du prêt } \\
\text { - Période durant laquelle la garantie peut être } \\
\text { - } \text { appelée } \\
\text { - Taux d'actualisation pour calculer la valeur } \\
\text { - Prime de réassurance }\end{array}$ \\
\hline \multicolumn{3}{|c|}{$\begin{array}{l}\text { * Dans l'hypothèse où l'instrument garanti est un prêt. } \\
\text { ** Dans le cas où une garantie couvre toute la durée du prêt, il n'est pas nécessaire de connaître la durée du prêt, la durée de la garantie et le taux } \\
\text { d'actualisation pour calculer le ratio de levier. Une variable est néanmoins nécessaire pour indiquer que l'on est dans ce cas. } \\
\text { ***Il se peut que l'institution garantisse les dernières années de remboursement du prêt. L'exposition de l'institution ne commence alors pas à la } \\
\text { signature du contrat, mais à la date à partir de laquelle la garantie peut être appelée. Dans ce cas, la valeur actuelle de l'exposition doit être calculée. }\end{array}$} \\
\hline
\end{tabular}

17. Par exemple, une exposition nette de 10 millions USD dans un PRI est généralement moins risquée qu'une exposition nette de 10 millions USD dans un PMA. 
